\title{
PER UNA TRADUZIONE MULTIDIMENSIONALE DELLA POESIA: \\ "NOTTE, STRADA, LAMPIONE, FARMACIA" DI ALEKSANDR BLOK \\ TRA ESISTENZIALISMO E SIMBOLISMO
}

\section{Carlo Testa*}

Poetry by definition is untranslatable.

Only creative transposition is possible.

Roman Jakobson (da Venuti 2000: 118; corsivo mio)

Quando leggo un testo tradotto di poesia vorrei poterlo leggere come fosse davvero un testo di poesia, cioè come testo in versi italiani plausibili. Il più delle volte invece ci si trova di fronte a quello che è in sostanza, avvertibilmente, un bizzarro residuo - più o meno dignitoso, ma in gran parte non poetico dell'originale, una sua generosa storpiatura.

Maurizio Cucchi (in Buffoni 1989: 93)

Riassunto: Questo articolo propone al pubblico di lingua italiana una nuova traduzione in verso e rima, commentata e ragionata, della classica poesia blokiana "Notte, strada, lampione, farmacia," anche nota come Danza macabra II (1912), tratta dalla raccolta Un mondo terribile (Strashnyi mir). La mia proposta viene presentata analiticamente attraverso un confronto puntuale con quattro preesistenti traduzioni in italiano: in primo luogo quella di Renato Poggioli, ne Il fiore del verso russo (1949), e successivamente quelle di Angelo Maria Ripellino (1975), Bruno Carnevali (1977) e Sergio Pescatori (1981). La caratteristica precipua di questa mia proposta è la presentazione di una traduzione poeti$c a$, ovvero una traduzione "multidimensionale" che nella lingua destinataria conservi, oltre ovviamente ad un massimo possibile della dimensione semantica (il "senso" strettamente letterale), anche tratti di verso e rima quanto più possibile simili a quelli che il testo di partenza possiede nella propria veste originale.

Al di là di alcune dettagliate, raffinate, ormai "classiche" analisi tecniche sulla natura della poesia cui ci hanno abituati i formalisti e gli strutturalisti ${ }^{1}$, nei termini più generali resta ancora vera l'apodittica formulazione data da Paul

* Desidero in questo frangente esprimere la mia gratitudine a tutti i colleghi - inclusi i lettori esterni di Quaderni d'Italianistica — che mi hanno preceduto: colleghi senza i cui sforzi (come afferma un luogo che non è men vero soltanto per essere comune) il mio lavoro non sarebbe davvero stato possibile.

1 Penso soprattutto a Brik (si veda Brik 1927/1968/1978) e Lotman (e.g. Lotman 1972/1976), ma tale campo è beninteso, ancor più che vasto, umom neizmirimo. 
Valéry all'essenza della questione: la poesia lirica, come si ricorderà, consiste per Valéry in "une hésitation prolongée entre le son et le sens"2. In altre parole, alla domanda "Come è fatta la poesia lirica?" (per parafrasare Šklovskii, che com'è noto esaminò di quale tessuto sia testo il Cappotto di Gogol, «kak sdelana Šinel' Gogol'ia»), l'inequivoca risposta di Valéry è che la poesia lirica "è fatta" (appunto, «sdelana») di valori semantici indistricabilmente intessuti con valori fonici (ritmo, rima: "melodia").

Tale è il cogito dal quale parto per riprendere la critica, da me già intrapresa tempo fa nel presentare una breve scelta di classici della poesia russa da me toscanizzati (Testa 2008, q.v.; è pronta a seguirla, in tempi tecnici, una mia versione dei Sonetti a Orfeo di Rilke), verso la prassi attualmente invalsa nell'industria editoriale italiana di offrire al pubblico italofono italologo — delle traduzioni strettamente monodimensionali, ovvero delle traduzioni unicamente semantiche, limitate al solo senso materiale dell'originale. Obietto, in altre parole, all'identificazione ormai data per scontata dall'industria editoriale "traduzione = traduzione letterale". Si intuirà come il concetto opposto, da me preferito, sia quello di una traduzione "totale," che io chiamerei multidimensionale 3 .

Lefevere, su cui elabora in dettaglio Bassnett (Bassnett 1980: 84-86), scrive a proposito della "literal translation" della poesia che in essa "the emphasis on word-for-word translation distorts the sense and the syntax of the original" (84). Tuttavia, dopo avere bene identificato un tale difetto, Lefevere ripreso da Bassnett passa poi a catalogare una lunga serie di ciò che chiamerei altri e diversi crimina interpretandi (crimini commessi riproducendo o "troppo suono", o "troppo metro", o "troppa prosa", o "troppa

2 Per la più dettagliata recente discussione della celebre "hésitation prolongée" rimando a Masseau, 40-43.

3 L'opposizione binaria qui messa a fuoco tra, da una parte, traduzione semantica / monodimensionale, e dall'altra, traduzione "totale," multidimensionale, inclusiva di valori fonetici / ritmici / cromatici / melodici / propriamente lirici, trascende i parametri del sia pur ottimo contributo di Sansone (in Buffoni 1989), il quale contributo discute "traduzione ritmica e traduzione metrica" ma nulla dice di una faccia altrettanto essenziale del poliedro poetico, la rima. Altrettanto poco di concretamente utilizzabile troviamo sull'argomento in Osimo 1998: 61. - A scanso di ogni fraintendimento metodologico, preciso che è soltanto una pura coincidenza se l'odierna teoria della traduzione si trova ad elaborare (teoricamente) sul concetto di "Multidimensional Translation" o "MuTra", che a quanto è dato comprendere teorizza su tipi di traduzione diagonali (multimediali) incrocianti svariati tipi di mezzi di comunicazione o artistici (teatro, cinema, opera ...). Si veda in proposito Heidrun Gerzymisch-Arbogast, 1-15. 
rima”, ecc.) senza purtroppo portare il ragionamento alla propria logica conclusione: alla conclusione, intendo dire, che la traduzione fra tutte meno inadeguata di una data poesia lirica sarà probabilmente quella che riuscirà a danneggiare quanto meno possibile l'originale, contemperando tutte le categorie suddette. Ma salvare l'aspetto lirico della lirica non sembra parere a Lefevere - Bassnett una preoccupazione degna di esame e discussione in quanto tale.

Ora, non vivo sulla luna ("I poeti devono, anche gli spirituali, / Appartenere al mondo..." $)^{4}$; mi rendo conto della complessità della questione da un punto di vista storico-culturale. Tempi grami per la lirica? Certo: esistono, per tali fenomeni, precisi contesti storici5. Per esempio, benissimo faceva Brecht a osservare — in una poesia degli anni di guerra appunto così intitolata, "Schlechte Zeit für Lyrik" — : "Nel mio canto una rima / Quasi mi parrebbe superbia ..."6 E subito ne spiega il perché: il sia pur forte desiderio del poeta di scrivere una felice, melodica poesia sull'amore e sulla natura deve cedere il passo all'urgenza di trovare, su ben diversi temi, ben più aspre parole, in un momento in cui l'Europa intera si trova a dover combattere gli orrori del nazifascismo.

Quindi, a guerra finita, ecco i letterati e poeti italiani giustamente lanciarsi a corpo morto sui grandi classici delle letterature moderne straniere ... precedentemente tenuti, dalla penisola, in gran dispitto ... per modernizzare e sprovincializzare.

Per de-estetizzare. Viva la de-estetizzazione!

Il problema è, però, che dopo il 1945 l'incautamente precipitosa e abbondante ingestione di traduzioni letterali e monodimensionali di - per esempio - Blok, Rilke e Pasternak portò i lettori italiani di poesia (che fino a prova contraria includono anche i poeti italiani, almeno si spera) a credere che i testi tradotti che essi leggevano fossero per l'appunto quelli di Blok, Rilke e Pasternak. Da ciò conseguiva che, nella mente di quei lettori e poeti italia-

${ }^{4}$ Die Dichter müssen auch die geistigen / Weltlich sein. Traduzione italiana mia. Vedi anche la trad. di Enzo Mandruzzato in Hölderlin, 649.

5 Il merito di avere introdotto la contestualizzazione storico-culturale nel mondo della traduttologia spetta, come è noto, a Lawrence Venuti, la conoscenza del cui nome e della cui invero meritoria opera da parte dei miei lettori davo, e do, per scontata. È meno certo che di una tale epifania critica si sentisse il bisogno nel campo degli studi letterari, ove la parola Kulturgeschichte circolava gà da tempo, anche senza traduzione.

6 In meinem Lied ein Reim / käme mir fast vor wie Übermut ("superbia” o "arroganza”). Mia traduzione. Vv. 15-16 di “Tempi grami per la lirica” ("Schlechte Zeit für Lyrik”), 1938-41. Brecht 1988: 14: 432. 
ni, per essere moderni e sprovincializzati bisognava scrivere come le traduzioni semantiche in italiano di - per esempio - Blok, Rilke e Pasternak. Nessuno tra essi pareva accorgersi che molti dei più grandi poeti europei del ventesimo secolo, additati a esempio per il rinnovamento della poesia italiana contemporanea, avevano scritto di regola in metro e rima.

Ecco dunque l'atroce mio dubbio: e se il rinnovamento della poesia italiana prodottosi nella seconda metà del ventesimo secolo fosse avvenuto, almeno in parte, sulla base di un errore di lettura? sulla base di un colossale abbaglio metodologico? Contesto storico finché si vuole, ma gli abbagli sono abbagli, punto 7 . O, detto in altri termini: un conto è de-estetizzare, tutt'altro è tradurre Rilke come se fosse Ezra Pound.

E tralascio del tutto di discutere qui il drammatico aspetto economicocommerciale della questione, che ci porterebbe troppo lontano ( $m a$ in proposito si trovano un'eccellente messa a fuoco in Sosio 1981, nonché svariati agghiaccianti faits divers, passim, in Buffoni 1989) ${ }^{8}$.

Messa in tal modo in chiaro l'importanza degli enjeux culturali di ciò che andiamo ad esaminare, nonché la nostra consapevolezza critica della "condizione storica" che vige nella repubblica letteraria, mi accosto ora al caso concreto di Blok e a quella che è forse la sua più celebre poesia. La caratteristica precipua di questa mia proposta è la presentazione di una traduzione poetica - una traduzione multidimensionale nel senso spiegato sopra - che nella lingua destinataria L2 conservi, oltre ovviamente ad un massimo possibile della dimensione semantica (il "senso" strettamente letterale), anche tratti di verso e rima quanto più possibile simili a quelli che

7 Per uno sviluppo più completo di tale argomento "culturale" si veda Testa 2008.

8 So benissimo che le imprese attive nel ramo dell'editoria italiana hanno dei vincoli di bilancio (nonché di profitto) ben precisi. D’altronde è proverbiale, lo si dice da millenni, che carmina non dant panem; quantomeno, ne danno poco ai loro traduttori. Ergo (e Bianciardi docet): se i traduttori dovessero lavorare senza fretta, non riuscirebbero affatto a campare. Goethe ebbe, per vie extra-editoriali, l'agio di scrivere una volta il Faust, ora, in tutta onestà quante volte potrebbero, i secoli a venire, permettersi di ri-finanziarne, sempre e di nuovo, la ri-traduzione con lo stesso agio che Goethe ebbe? Lo ammetto: nel tradurre monodimensionalsemanticamente si lavora molto più a spron battuto che nel tradurre multidimensionalmente in ritmo e rima. Lo ammetto, l'industria editoriale ha ritmi (anche se non rime ...) che non tollerano eccessivi perfezionismi. Tuttavia, la pecca non cessa di essere tale soltanto perché si rivela funzionale ai bisogni commerciali di un'industria che, come di ogni industria si suol dire, "crea occupazione," ecc. - Sulla teoria della funktionsgerechte Übersetzung, che quivi proietta la sua ombra, si veda infra la discussione circa Snell-Hornby 2006 (mia nota 10). 
l'originale possiede nella propria lingua L1. Superando le prestazioni delle traduzioni condotte nello spazio unicamente monodimensionale della sola semantica, il principio della traduzione poetica in verso e rima sembra a chi scrive essere infatti sempre rilevante in tutti i tipi di poesia lirica, ma addirittura fondamentale, fondante, indispensabile nel caso della lirica simbolista, di cui Blok è uno dei massimi maestri`.

Nel concreto, come caso esemplare di "atelier del traduttore" questo articolo propone al pubblico di lingua italiana una nuova traduzione, commentata e ragionata, della classica poesia blokiana "Notte, strada, lampione, farmacia," nota anche come "Danza macabra II" ("Pliask smerti II," del 10-10-1912), tratta dalla raccolta Un mondo terribile (Strašnyi mir, 19091916; in Polnoe Sobranie Stixotvorenii, \# 539, vol. 1, pp. 376-77). La mia proposta viene presentata analiticamente attraverso un confronto puntuale con quattro preesistenti traduzioni in italiano: in primo luogo quella di Renato Poggioli, ne Il fiore del verso russo (1949; trad. a p. 278), e successivamente quelle di Angelo Maria Ripellino (1975), Bruno Carnevali (1977), e Sergio Pescatori (1981).

Come è noto a chi ne conosce la meritoria opera - profetica e, al tempo, poco e mal compresa - Poggioli stesso fu ovviamente un araldo della traduzione poetica che ho appena descritto come multidimensionale. Il problema, tuttavia, è che Poggioli talvolta giunse ai propri risultati prendendosi delle libertà semantiche, a mio avviso, maggiori di quanto sarebbe stato ottimale o, in ogni caso, necessario.

Ripellino e Carnevali ci offrono invece degli esempi sintomatici del metodo opposto: vale a dire, delle traduzioni monodimensionali che sono (o, almeno, quasi sempre sono) "sufficienti" a soddisfare i nostri bisogni semantici, ma che per ciò fare massacrano il lato materialmente e tangibilmente armonico della lirica originale, fino al punto di rendere se stesse quasi del tutto inservibili, se non in funzione puramente informativa, per chi non conosca abbastanza il russo per andarsi a leggere da sé il testo di Blok. A un tale punto, paradossalmente, diviene persino difficile parlare di una "traduzione" in qualunque senso efficace: se per gustare la tangibilità concreta dell'arte di Blok dobbiamo essere noi stessi in grado di appercepire l'originale - allora, delle traduzioni "informative" come quelle redatte da Ripellino e Carnevali che ce ne facciamo? Poco; a meno che, si capisce,

9 Pongo dunque una assoluta equivalenza concettuale "traduzione (pienamente) poetica = traduzione multidimensionale" - traduzione, cioè, che tenga conto delle multiple facce stereometriche di un dato e concreto oggetto-poesia. 
"...unless the translation is intended as a crib", "a meno che la traduzione venga intesa come un bigino [semantico]" (per trasportare a questo contesto Bassnett 2002: 86) ${ }^{10}$. Altrove si assegna una positiva funzione, se non altro, ancillare a tali cosiddette "traduzioni di servizio"; ma anche questo concetto è problematico ${ }^{11}$.

10 E ancora. Quale potrebbe essere, per esempio, lo scopo di un ipotetico "bigino" strettamente semantico di "Nulla, questa schiuma, vergine verso / Da non designare che la coppa; / Tale, lontano, annega una truppa / Di sirene, qualcuna all'inverso. // Noi navighiamo ...," ecc. ecc.? oppure di: "Intendi, mia cara, intendi la dolce Notte che marcia"? È evidente che la "svolta" di Translation Studies verso lo "skopos" o "funzionalità" descritta in Snell-Hornby (51-56 e passim) non ci aiuta minimamente nel contesto della lirica. Sembra infatti corretto, ma anche vuotamente tautologico, asserire che "the one and only perfect translation does not exist, any translation is dependent on its skopos and its situation" (52); "a translation is seen in terms of how it serves its intended purpose"; [la traduzione è] "ein Informationsangebot in einer Sprache $z[\ldots]$, das ein Informationsangebot in einer Sprache a funktionsgerecht imitiert» (53, corsivi miei). Alla buon'ora, quando si tratti di "the instructions for use of a washing machine" (58)! Ma quando si desideri invece rendere, per il beneficio dei propri lettori italiani, la sorcellerie évocatoire contenuta in una lirica di (per esempio) Blok, a che criterio potremo affidarci per stabilire con certezza che il fattore in essa tra tutti dominante (jakobsonismo qui da me evidentemente voluto), e dunque più di tutti funktionsgerecht ai fini della traduzione da intraprendersi, dovrà essere il suo aspetto semantico a preferenza di quello melodico? Ben si vede come la "teoria funzionale" sia incapace di dimostrare in modo razionalmente fondato, nel campo della poesia lirica, la presunta supremazia della semantica. Non sappiamo forse che "testo poetico è un testo caratterizzato principalmente da significati connotativi" (Osimo 1998: 14)? - A meno che lo skopos concettuale sotteso a una tale mossa (accanto a quello commerciale della massimizzazione dei profitti, cui ho già accennato e su cui dunque non torno) non sia invece di scrivere traduzioni che si esibiscano — alla maniera dell'"esibirsi" degli acrobati appunto come traduzioni: ovvero, contengano quel tanto di innaturale e forzato che ce le faccia all'istante identificare per tali (la famosa nozione di deliberata "foreignizing translation" venutiano). Ha senso una tale proposta per la lirica il cui originale è scritto in verso e rima? In un tale spirito, altrettanto potrebbero i musei del mondo, a scanso di "troppo levigate" e dunque "pericolose" illusioni di realtà, decidere di mettere al bando le riproduzioni a colori (cromatiche) delle proprie opere d'arte.

11 'Molte 'traduzioni' 'in versi' (fra virgolette ripetute, perché in verità tali non sono nell'uno e l'altro caso) si propongono, quasi a titolo di merito, come non sostitutive del testo originale, [bensì come] una sorta di sussidio per chi non conosce a sufficienza la lingua da cui si traduce, fornendo una possibile inter- 
Pescatori si dimostra conscio di tali problemi, in parte li esamina, e propone poi una sua traduzione alternativa; la quale però presenta anch'essa, accanto a soluzioni interessanti, alcuni aspetti spinosi, che illustrerò.

La mia traduzione che verrà qui proposta mira dunque, pur riconoscendo i meriti individuali di chi mi ha preceduto, a trovare un'ideale aurea mediocritas tra i due estremi metodologici testé delineati, non molto diversa nella teoria dalla proposta di Pescatori ma con alcune varianti pragmatiche alternative, che spiegherò a tempo debito.

\section{Blok e la via senza uscita}

Preliminarmente: perché scegliere "Notte, strada, lampione, farmacia"? Ebbene, la seconda delle due danze macabre di Blok gode di tre notevoli vantaggi:

I), è riconosciuta dai critici come una delle più riuscite opere del poeta (e difatti è forse la sua poesia più frequentemente tradotta in varie lingue);

II), esiste in più versioni italiane pubblicate, permettendo con ciò un'utile analisi comparativa e differenziale di pratiche concrete di traduzione / trasposizione poetica; e

III), grazie alla sua concisione e alla sua struttura circolare possiede una potente qualità aforistica che si imprime con facilità nella mente dei lettori.

Un'ulteriore fonte di interesse di questa poesia - quanto meno per i comparatisti - è che stabilisce un'evidente tensione dialogica con il ben noto (e forse troppo ottimista ...) detto di Goethe "Stirb und werde", "Muori e divieni."

Ecco dunque la poesia di Blok:

Ночь, улица, фонарь, аптека, бесмысленный и тусклый свет.

Живи еще хоть четверть века -

всё будет так. Исхода нет.

Умрешь - начнешь опять сначала,

pretazione senza sovrapporsi al testo. A parte il fatto che per intenderla una lingua non ammette scarsità di conoscenza, [...] l'infedeltà di questi tentativi riesce a volte persino patetica, gettando il lettore nella costernazione di non riconoscere e intendere nemmeno la propria lingua [...] Se manca la volontà precisa di ricostruire in un altro sistema linguistico, con gli ingranaggi poetici che gli sono propri, la tensione dell'originale, l'operazione non solo non ha rilievo criti$\mathrm{co}, \mathrm{ma}$ è irrimediabilmente perduta, perfettamente inutile. L'inganno nasce dalla disperata speranza che il segno sia univoco, non abbia risvolti, non significhi altro che se stesso". Ramous in Buffoni 230. E ancora: "La fedeltà limitata al senso è una forma di tradimento". Valéry in Osimo 60. 
и повторится всё, как встарь:

ночь, ледяная рябь канала,

аптека, улица, фонарь.

Come nella maggior parte della sue poesie, in "Pliask smerti II" Blok si serve del tetrametro giambico — dai tempi di Pushkin, il verso classico della poesia russa - in varianti che alternano parole piane / parossitone, dall'accento posto sulla penultima sillaba, con parole tronche / ossitone. Se applicato inflessibilmente, tuttavia, un tale schema può risultare terribilmente monotono, e nessun poeta vorrebbe mai aderirvi in modo acritico.

Al fine di evitare l'imitazione del ritmo ripetitivo di un treno in corsa, o peggio, di un'istupidente marcetta militare — due possibili cattivi esiti dell'uso del ritmo binario in poesia - i poeti russi, in pratica, sistematicamente introducono dunque delle variazioni nello schema teorico puramente binario dei loro giambi: lasciano non accentate alcune sedi teoricamente accentande, anzi talvolta invertono la posizione delle sillabe accentate con le non accentate, creando, al lume della loro ispirazione, una metrica "sbagliata."

Si potrebbe addirittura dire che in russo lo scopo stesso della versificazione (come della lettura critica di essa) consiste nell'analizzare e comprendere dove, come e perché la prassi poetica devii da un dato schema previsto, ma non perciò prescritto.

I formalisti russi ci hanno insegnato che la distinzione tra il linguaggio quotidiano e il linguaggio letterario, dunque a fortiori il linguaggio poetico, consiste nelle deviazioni che quest'ultimo compie rispetto ad una certa norma prestabilita, ovvero nella probabilità sempre più ridotta delle associazioni di parole che esso offre ad ognuno dei suoi successivi stadi semantici. Lo stesso principio può facilmente essere applicato alla versificazione russa, ove l'ictus "eccezionale" introdotto in un ritmo che empiricamente si discosti dal suo astratto prototipo sembra, a parere dei critici, aumentare la specificità / quantità di informazione ("poeticità") di un dato testo poetico e renderlo - entro certi parametri — per così dire doppiamente poetico.

Una tale deviazione dal modello giambico è appunto ciò che ritroviamo in "Pliask smerti II." Soltanto i versi 3 e 5 sono giambi regolari, accentati sulle sillabe regolari 2, 4, 6 e 8 . Invece i versi 1 e 2 perdono il secondo dei loro accenti canonici, previsto sulla sillaba 4; ai versi 6 e 7 manca il primo accento, teoricamente previsto sulla sillaba 2 ; e il verso 8 perde il suo terzo ictus, che avrebbe dovuto cadere sulla sillaba 6. Insomma, gettando anche solo un primo sguardo analitico sulla prosodia blokiana si rileva che, invece di decidere a priori con quale ritmo di verso italiano si debba, o meno, rendere dei versi originali russi, ci incombe il considerare che non è 
assolutamente detto che quegli originali siano essi stessi schematicamente uniformi. Ci si deve anzi assolutamente attendere che non lo saranno; e che quindi non soltanto consentiranno, in italiano, una variabilità ritmica corrispondentemente flessibile, ma che addirittura la inviteranno.

Ancor più rivelatori delle posizioni ritmiche lasciate non occupate in "Pliask smerti II" sono, mi sembra, i casi inversi: le posizioni, intendo, che pur dovendo in teoria essere senza accento, in pratica ricevono invece un forte ictus. Vi sono tre casi di tal genere in questa poesia di Blok, e ognuno di essi riguarda la prima sillaba di un verso (ovvero i versi 1, 4 e 7). Questo non è un punto meramente tecnico. Per comprenderne il motivo, basta che esaminiamo il lato semantico del tessuto della poesia: come si può notare, quelle tre posizioni sono occupate da monosillabi vigorosi, carichi di un ruolo cruciale nell'argomento svolto dal poeta.

Così il verso 4 ci colpisce di primo acchito con la parola vsë, "tutto" ("tutto sarà eguale. Non c'è uscita"). Per parte loro i versi 1 e 7 , come a voler materializzare il buio spaesamento, sia fisico che metaforico, esperito dall'io lirico, scandiscono fortemente la loro prima parola, noč" ("Notte, strada, lampione, farmacia"; "la notte, il gelido incresparsi del canale"). La posizione eccezionale di questi tre nodi ritmico-semantici indica con chiarezza che la non completa uniformità del ritmo è il concetto essenziale da cui partire per il nostro esercizio di interpretazione e traduzione.

\section{Poggioli: il ritmo}

Inizierò la discussione presentando per prima la traduzione di Poggioli $(F V R$, p. 278), che è il traduttore italiano cui va il merito di avere quanto meno aperto la strada del pubblico italiano e italofono verso un patrimonio letterario precedentemente riservato agli slavisti.

Ecco la versione poggioliana:

Un melanconico riflesso

— strada lampione farmacia -

è fu sarà sempre lo stesso -

non c'è più scampo: così sia.

E vita e morte, e bene e male

ritrovi ad ogni crocevia:

è notte - ghiaccio sul canale -

strada lampione farmacia.

Poggioli sceglie di rendere l'originale di Blok, redatto in novenari alternati tra piani e tronchi, con dei novenari italiani. Questa è una sfida notevole per qualunque traduttore italiano, dato che la lingua russa - come la lati- 
na, ma a differenza della italiana - è estremamente sintetica e metricamente flessibile, ricca di parole (nomi, aggettivi, pronomi, avverbi, forme verbali) tronche o addirittura monosillabiche. Lunica attenuazione che Poggioli si concede nel mettersi in tal modo alla prova è di rinunciare a produrre parole italiane tronche per concludere i versi pari 2, 4, 6, 8, in corrispondenza di (rispettivamente) svet, net, vstar' e fonar': i suoi novenari rimangono coerentemente piani (cioè hanno tutti nove sillabe, con ogni penultima sillaba regolarmente accentata) - e la sua traduzione può così evitare l'effetto di artificialità, o condizionatezza storica, che in italiano spesso caratterizza l'uso delle parole tronche, per esempio nei libretti d'opera. In compenso però Poggioli complica la propria missione con il secondo vincolo di cui decide di farsi carico, il quale consiste nella riproduzione, nella propria traduzione di "Pliask smerti II," di un ritmo giambico non meno rigoroso (anzi di più, come vedremo tra un momento) rispetto a quello originale.

Poggioli raggiunge il proprio scopo ritmico con notevole sagacia. I suoi ritmi giambici sono regolari: addirittura più regolari di quelli di Blok stesso. (Ghini nota lo stesso fenomeno nelle traduzioni poggioliane da Pushkin; Ghini 2005: 89). I versi di Poggioli 5, 6 e 7 sono tetrametri giambici da manuale, con gli accenti disposti sulle sillabe 2, 4, 6, 8:

$\begin{array}{ll}\text { POG 5 } & \text { E VI } \mid \text { ta e MOR } \mid \text { te, e BE } \mid \text { ne e MA le } \\ \text { POG 6 } & \text { ri TRO } \mid \text { vi ad O } \mid \text { gni CRO } \mid \text { ce VI a: } \\ \text { POG } 7 & \text { è NOT } \mid \text { te }- \text { GHIAC } \mid \text { cio SUL } \mid \text { ca NA le - }\end{array}$

Inoltre i versi 1 e 4 mostrano una minima deviazione dalla regola, nella forma di una 6a sillaba non accentata:

$\begin{array}{ll}\text { POG } 1 & \text { Un ME } \mid \text { lan CO } \mid \text { ni }<\text { co }>\mid \text { ri FLES so } \\ \text { POG } 4 & \text { non C'È } \mid \text { più SCAM } \mid \text { po: }<\text { co }>\mid \text { sì SI a }\end{array}$

Una reale divergenza di Poggioli dal ritmo giambico rigidamente inteso si verifica soltanto nei versi 2 ed 8 "strada lampione farmacia," vale a dire:

POG $2+8 \quad$ STRA da $\mid$ lam PIO $\mid$ ne FAR $\mid$ ma CI a

ove la distribuzione degli accenti sulle sillabe 1 e 2 è invertita, producendo così lo scontro fra due sillabe non accentate consecutive, la 2 e la 3 . Ma naturalmente, dato il contenuto semantico del verso finale di Blok apteka, ulitsa, fonar' - sarebbe improbo riuscire a rendere l'originale con un novenario italiano il cui dettato fosse diverso da quello scelto (o subìto) da Poggioli. 


\section{Poggioli: il significato}

Esaminiamo ora come il lato semantico di Blok sia, o meno, accolto nella versione poggioliana di "Pliask smerti II." Considererò più avanti le altre traduzioni in italiano.

Per la prima metà della poesia annotiamo la seguente sinossi interlineare:

\begin{tabular}{|c|c|}
\hline $\begin{array}{l}B L O K 1 \\
\text { POG } 1\end{array}$ & $\begin{array}{l}\text { Ночь, улица, фонарь, аптека, } \\
\text { Un melanconico riflesso }\end{array}$ \\
\hline $\begin{array}{l}\text { BLOK } 2 \\
\text { POG } 2\end{array}$ & $\begin{array}{l}\text { бесмысленный и тусклый свет. } \\
\text { - strada lampione farmacia - }\end{array}$ \\
\hline $\begin{array}{l}B L O K 3 \\
\text { POG } 3\end{array}$ & $\begin{array}{l}\text { Живи еще хоть четверть века } \\
\text { è fu sarà sempre lo stesso - }\end{array}$ \\
\hline $\begin{array}{l}B L O K 4 \\
\text { POG } 4\end{array}$ & $\begin{array}{l}\text { всё будет так. Исхода нет. } \\
\text { non c'è più scampo: così sia. }\end{array}$ \\
\hline
\end{tabular}

Benché l'alchimia versificatoria di Poggioli sia complessa, le sue conseguenze semantiche non sono difficili da discernere. All'inizio, il senso "Notte via lampione farmacia" è stato rimosso e spostato dal verso 1 al verso 2 , nella forma "strada lampione farmacia"; insomma, il primo verso poggioliano ha sofferto l'ablazione della primissima parola, l'incipit "Noč ...". È un problema per chi volesse mai reperire il testo, per esempio, in qualche edizione in lingua originale. Per fortuna questa poesia è così famosa che risulta difficile immaginare come chiunque la cerchi in un'edizione in lingua russa possa non sapere quale sia davvero la prima parola scritta da Blok.

E che troviamo, dunque, nel verso 1 evacuato da Poggioli? Troviamo "Un melanconico riflesso": ovvero il presunto equivalente del verso 2 dell'originale, "una luce insensata e fioca." Però "un melanconico riflesso" è una resa fortemente interpretativa - e, oltretutto, una resa che complica considerevolmente la questione. Nell'originale, "insensata e fioca" possiede infatti una brutalità fattuale che trasmette in modo diretto una quasi tramortita mancanza di elaborazione da parte della voce poetica; per contro, l'uso che Poggioli fa della parola "melanconico" suggerisce una consapevolezza metacritica, forse perfino un certo stoico distacco, rispetto alla propria - e nostra universale - condizione umana.

Ulteriori divergenze ci attendono nei versi 3 е 4. Nel verso 3, “Живи еще хоть четверть века," "Se anche vivessi ancora un quarto di secolo," diviene in Poggioli "è fu sarà sempre lo stesso." Tale formulazione 
(anche a prescindere dalla innecessaria confusione causata dall'assenza di interpunzione) è, come ben si vede, una lontana parafrasi dell'originale; ed è, oltretutto, una parafrasi che va a rosicchiare in anticipo parte del contenuto semantico a venire nel verso seguente. Ciò senza contare che nell'originale il poeta presenta l'ipotesi di poter / dover vivere un altro quarto di secolo come una sventura non meno insensata e squallida della luce che imbeve l'ambiente da cui si sente oppresso. Un così personale senso di calamità manca nelle parole italiane "è fu sarà sempre lo stesso," che sono sì algide, ma in un modo cerebrale del tutto in contrasto con il freddo, fisicamente penetrante fino al midollo, della Pietroburgo notturna di Blok.

Anche il verso 4 porta delle sorprese, qui sotto forma di creazione discorsiva. "Всё будет так. Исхода нет," "Tutto sarà così. Non c'è uscita," diviene per Poggioli "Non c'è più scampo: così sia," che una volta di più implica un allontanamento considerevole dal senso dell'originale, in ognuno dei suoi concetti. Perché più - un termine che sembra suggerire che "prima" le cose stessero diversamente? A che pro l'enfatico scampo, anziché l'alternativa corretta - e drammaticamente semplice - della parola usci$t a$ ? (Uscita è metricamente equivalente a scampo, in questo verso almeno. Tornerò più tardi sull'opzione esodo, suggerita da Ghini). E perché poi cosi sia, con la sua eco che in italiano è prevalentemente religiosa? Forse la spiegazione è semplicemente che Poggioli non avesse sottomano una miglior rima con cui accompagnare farmacia? (Similmente si osserva, seppure in modo più allusivo, in Pescatori 1981: 86).

Passiamo ora alla seconda parte della poesia:

$\begin{array}{ll}\text { BLOK 5 } & \text { Умрешь - начнешь опять сначала, } \\ \text { POG } 5 & \text { E vita e morte, e bene e male } \\ \text { BLOK 6 } & \text { и повторится всё, как встарь: } \\ \text { POG 6 } & \text { ritrovi ad ogni crocevia: } \\ \text { BLOK } 7 & \text { ночь, ледяная рябь канала, } \\ \text { POG } 7 & \text { è notte - ghiaccio sul canale - } \\ \text { BLOK 8 } & \begin{array}{l}\text { aптека, улица, фонарь. } \\ \text { POG } 8\end{array}\end{array}$

I versi 5 e 6 vanno chiaramente al cuore dell'angoscia vissuta dal poeta. "Умрешь - начнешь опять сначала, / и повторится всё, как встарь": "Morirai, e ricomincerai daccapo, / e tutto si ripeterà come in passato." Qui i versi corrispondenti di Poggioli, di cui ho già lodato la capacità di fondere impeccabilmente il novenario italiano con il ritmo giambico dell'originale, mostrano l'alto - l'astronomico, si potrebbe dire - 
costo semantico del voler concedere al ritmo una priorità assoluta rispetto al significato. Si nota infatti a prima vista come le parole "E vita e morte, e bene e male / ritrovi ad ogni crocevia," nel concreto non abbiano purtroppo granché in comune con la semantica dei versi 5 e 6 di questa poesia di Blok.

Non saprei citare un altro caso suscettibile di mostrare più chiaramente come il processo di traduzione consista in ultima analisi in un tipo speciale di assegnazione di priorità. Non esiterei a metaforizzare la traduzione (e segnatamente la traduzione poetica) con l'immagine di un procedimento di compromesso economico: le risorse sono limitate, e tali sono anche soprattutto in poesia - le opzioni combinatorie di un qualunque testo dato $^{12}$. Ne consegue che le risorse profuse nell'intento di privilegiare un dato aspetto di una data transcodificazione per ciò stesso non saranno più disponibili per "co-privilegiare" altre transcodificazioni in concorrenza con la prima. Non si può privilegiare tutto ${ }^{13 !}$

Nei versi 7 e 8 , che concludono la poesia, il genio poetico di Blok porta i suoi frutti. Rovesciando l'empito nietzscheano verso un liberatorio futuro circolare, l'eterno ritorno temuto dalla voce poetica di Blok diviene ora una profezia adempiuta. Un tale cosmico ritorno si avvera nel preciso momento - nell'atto stesso - in cui il poeta, con minime varianti, ripete le parole già usate nel v. 1: "ночь, ледяная рябь канала, / аптека, улица, фонарь" - "la notte, il ghiacciato incresparsi del canale, / la farmacia, la via, il lampione."

E così, grazie alla sorcellerie évocatoire di Blok, circolare nonché lievemente autodistruttiva, nel momento in cui inizia l'ultimo verso della poe-

12 Per la traduzione poetica come “compromesso" si veda Newmark, cit. in Sansone 17, n. 10.

13 Come nella vita anche nella traduzione, da qualche parte, in qualche modo, bisogna in definitiva scegliere. Sull'argomento si veda Levý, "Translation as a Decision Process," in Venuti 148-59. Ancora più chiaramente viene messo l'accento sulla traduzione come sequenza proairetica di scelte da Giovanni Giudici: "In fondo, il lavoro del traduttore di poesia si configura come una serie o successione di scelte, una serie o successione di costrizioni a rinunciare a qualcosa che è nell'originale e che non potrà essere nella traduzione se non al prezzo di sacrificare qualche altro valore di senso ancora più importante e magari decisivo, perché una certa 'essenzialità' o 'tipicità' dell'originale sia in qualche modo veicolata dalla traduzione. Ma è proprio nell'affrontare certe scelte e nel passare attraverso le forche caudine di certe costrizioni che si definisce l'opera del traduttore di poesia ..." ecc. (Giudici in Buffoni 85). 
sia i lettori hanno già sviluppato un fondato presentimento circa il modo in cui essa finirà. Si potrebbe anzi dire che ciò che rende davvero perfetta "Danza macabra II" è proprio il fatto che noi, invece di coglierne con i nostri sensi (leggerne o udirne) il finale, precorriamo quella chiusa attraverso un'intuizione della nostra mente; e poiché, ciò facendo, precediamo di una frazione di secondo i nostri sensi sul traguardo della comprensione, proprio grazie a tale effetto ri-conosciamo nelle ultime parole del poeta qualcosa di cui già avevamo avuto precognizione. E una tale precognizione è precisamente ciò a cui questa poesia fin dall'inizio mirava.

In tali circostanze, nel tradurre i versi 7 e 8 non poteva certo porsi il caso di una resa in italiano, per quanto semanticamente esuberante, che osasse allontanarsi molto dal sentiero tracciato dall'originale. E dunque, Poggioli scrive: " $\grave{E}$ notte — ghiaccio sul canale — / strada lampione farmacia." In primo luogo, la sintassi poggioliana non è lineare: in Blok, ognuno dei nomi elencati è un'apposizione alla parola vsë, "tutto"; Poggioli invece applica a notte un'asimmetrica copula che non vale per i nomi seguenti, e abbandona questi ultimi al loro destino. Inoltre, la parola notte non figura nel primo verso di Poggioli (id est, nel secondo verso di Poggioli); e dunque, nella traduzione poggioliana l'universale ritorno del passato non si applica alla notte, risultando con ciò meno che universale.

Ovviamente, le complicazioni che vediamo insorgere nella chiusa di Poggioli sono dei "debiti" da lui contratti in conseguenza di scelte precedenti: vale a dire, la sintassi problematica dei vv. poggioliani 5 e 6 , nonché il dettato, quantomeno aggrovigliato, scelto da Poggioli per i vv. 1 e 2. Una scelta di rime sub-ottimale (dovuta, si suppone, al fatto che il ritmo riceve qui la priorità assoluta) ha probabilmente aggravato il peso di un tale passivo.

\section{Ripellino e Carnevali, commentati e in parte migliorati da Pescatori}

Avendo così stabilito un punto di partenza storico, passiamo ora ad esaminare due alternative successive: le due traduzioni eseguite rispettivamente da Angelo Maria Ripellino (pubbl. 1975) e Bruno Carnevali (pubbl. 1977).

Ecco la versione di Ripellino:

$\begin{array}{ll}\text { RIP } 1 & \text { Notte, strada, fanale, farmacia, } \\ \text { RIP 2 } & \text { una luce assurda ed appannata. } \\ \text { RIP } 3 & \text { Pur se ancora vivrai venticinque anni - } \\ \text { RIP 4 } & \text { sarà sempre cos̀. Non c'è rimedio. } \\ \text { RIP 5 } & \text { Tu morirai — comincerai di nuovo, }\end{array}$




$\begin{array}{ll}\text { RIP } 6 & \text { e tutto riaccadrà come una volta: } \\ \text { RIP } 7 & \text { gelido incresparsi del canale, } \\ \text { RIP } 8 & \text { notte, farmacia, strada, fanale. }\end{array}$

Segue poi la proposta di Carnevali:

CAR 1 Notte, fanale, strada, farmacia,

CAR 2 luce assurda, appannata.

CAR 3 Vivessi ancora un quarto di secolo,

CAR 4 non cambierebbe nulla. Senza scampo.

CAR 5 Muori e rinasci ancora,

CAR 6 e tutto sarà uguale come prima:

CAR 7 freddo incresparsi del canale, notte,

CAR 8 strada, fanale, farmacia.

Sergio Pescatori (1981) propone quanto segue (tra breve metterò in parallelo le varie alternative, riconoscendo il valore dei suggerimenti di Pescatori):

$\begin{array}{ll}\text { PES 1 } & \text { Notte, strada, fanale, farmacia, } \\ \text { PES } 2 & \text { un'assurda luce sbiadita. } \\ \text { PES 3 } & \text { E per quanti anni la sorte ti dia - } \\ \text { PES 4 } & \text { così per sempre, senza uscita. } \\ \text { PES 5 } & \text { Morrai; poi ricomincerai daccapo, } \\ \text { PES 6 } & \text { e tutto sarà ancora uguale: } \\ \text { PES 7 } & \text { notte, un brivido sul canale gelato, } \\ \text { PES } 8 & \text { farmacia, strada, fanale. }\end{array}$

Sembra utile, a questo punto, procedere ad un sorvolo complessivo, rigo per rigo, delle quattro possibilità di traduzione citate finora:

$\begin{array}{ll}\text { BLOK 1 } & \text { Ночь, улица, фонарь, аптека, } \\ \text { POG 1 } & \text { Un melanconico riflesso } \\ \text { RIP 1 } & \text { Notte, strada, fanale, farmacia, } \\ \text { CAR 1 } & \text { Notte, fanale, strada, farmacia, } \\ \text { PES 1 } & \text { Notte, strada, fanale, farmacia, } \\ \text { BLOK 2 } & \text { бесмысленный и тусклый свет. } \\ \text { POG 2 } & \text { - strada lampione farmacia - } \\ \text { RIP 2 } & \text { una luce assurda ed appannata. } \\ \text { CAR 2 } & \text { luce assurda, appannata. } \\ \text { PES 2 } & \text { un'assurda luce sbiadita. } \\ \text { BLOK 3 } & \text { Живи еще хоть четверть века - } \\ \text { POG 3 } & \text { ѐ fu sarà sempre lo stesso - }\end{array}$




\begin{tabular}{|c|c|}
\hline RIP 3 & Pur se ancora vivrai venticinque anni - \\
\hline CAR 3 & Vivessi ancora un quarto di secolo, \\
\hline PES 3 & E per quanti anni la sorte ti dia - \\
\hline $\begin{array}{l}B L O K 4 \\
\text { POG } 4 \\
\text { RIP } 4 \\
\text { CAR } 4 \\
\text { PES } 4\end{array}$ & $\begin{array}{l}\text { всё будет так. Исхода нет. } \\
\text { non c'è più scampo: соsì sia. } \\
\text { sarà sempre così. Non c'è rimedio. } \\
\text { non cambierebbe nulla. Senza scampo. } \\
\text { così per sempre, senza uscita. }\end{array}$ \\
\hline & $*$ \\
\hline$B L O K 5$ & Умрешь - начнешь опять сначала, \\
\hline POG 5 & E vita e morte, e bene e male \\
\hline RIP 5 & Tu morirai - comincerai di nuovo, \\
\hline CAR 5 & Muori e rinasci ancora, \\
\hline PES 5 & Morrai; poi ricomincerai daccapo, \\
\hline$B L O K 6$ & и повторится всё, как встарь: \\
\hline POG 6 & ritrovi ad ogni crocevia: \\
\hline RIP 6 & e tutto riaccadrà come una volta: \\
\hline CAR 6 & e tutto sarà uguale come prima: \\
\hline PES 6 & e tutto sarà ancora uguale: \\
\hline$B L O K 7$ & ночь, ледяная рябь канала, \\
\hline POG 7 & è notte - ghiaccio sul canale - \\
\hline RIP 7 & gelido incresparsi del canale, \\
\hline CAR 7 & freddo incresparsi del canale, notte, \\
\hline PES 7 & notte, un brivido sul canale gelato, \\
\hline$B L O K 8$ & аптека, улица, фонарь. \\
\hline POG 8 & strada lampione farmacia. \\
\hline RIP 8 & notte, farmacia, strada, fanale. \\
\hline CAR 8 & strada, fanale, farmacia. \\
\hline PES 8 & farmacia, strada, fanale. \\
\hline
\end{tabular}

\section{Verso 1}

Nel v. 1, con spirito letterale Ripellino scrive "Notte, strada, fanale, farmacia"; Carnevali, nell'intento di differenziare la propria sequenza, inverte la via con il lampione e decide in favore di "Notte, fanale, strada, farmacia." Pescatori duplica Ripellino. Perché, in tutti e tre i casi, "fanale"? Il "lampione" poggioliano sembra molto più persuasivo.

A questo proposito, Pescatori argomenta per una ipotetica "scuola del fanale" affermando che in italiano fanale è parola letteraria, mentre lampione sarebbe "poco 'poetico" (Pescatori 1981: 84). Forse; non ho le qua- 
lifiche per entrare in un tale dibattito. Ma usiamo la macrovisione anziché la microvisione: è, a sua volta, "poetica" la parola farmacia? Non si direbbe; eppure nessuno la contesta, o men che meno la rimpiazza con altra più aulica. No: è chiaro come in questa poesia Blok miri precisamente a sottolineare l'allucinante stranezza, lo žutko (das Unheimliche) evocati dal quotidiano. Rovesciando l'idealismo magico à la Novalis e à la Eichendorff, in cui "il mondo prende a cantare" (die Welt hebt an zu singen, secondo la formula eichendorffiana), qui si può ben dire che improvvisamente "il mondo prende a stridere," a deformarsi negli spettri di un Romanticismo ereditato da E.T.A. Hoffmann: gli spettri di cose banali e prosaiche, come le farmacie ... e con esse, appunto, i lampioni.

Per queste ragioni prenderò qui dunque le parti di Ripellino, e proporrò semplicemente

TES 1

Notte, strada, lampione, farmacia,

Questa traduzione è semanticamente precisa, e dà un verso italiano non troppo sgraziato (un endecasillabo fortemente ritmato).

\section{Verso 2.}

Ripellino: "una luce assurda ed appannata." Carnevali: "luce assurda, appannata." Pescatori: "un'assurda luce sbiadita." In primo luogo, Carnevali mostra di avere scarsa inventiva e nessun senso del ritmo (o, se vogliamo, molto senso del ritmo, applicato ad evitare ogni ritmo purchessia nella sua traduzione). Ma continuiamo. Inoltre, sembra improprio l'uso in tutte e tre le versioni - del termine assurdo. Bisogna ammettere che, per chiunque giunga non troppo tempo dopo Sartre, Camus e Ionesco, l'idea di infilare la parola assurdo nelle scarpe di Blok possa avere una certa attrattiva, quantomeno dal punto di vista divulgativo. Ho però l'impressione che si tratti di un anacronismo, da quantificarsi probabilmente con una cifra compresa tra i venticinque e i trent'anni: La nausée fu pubblicata soltanto nel 1938. Comunque, e soprattutto: besmyslennyi non significa "assurdo" (che in russo si dice nelepyi; la littérature de l'absurde è poi, con logico terminus technicus, литература абсурда, literatura absurda), bensì precisamente insensato (fr. insensé, ted. sinnlos - di cui si può supporre che besmyslennyi sia un calco). Infine, le parole "appannata" e "sbiadita," pur accettabili come equivalenti di tusklyi, non sono particolarmente potenti, palpitanti, evocative; presentano per soprammercato lo svantaggio di portarci in un vicolo cieco per quanto concerne la nostra ricerca di rime italiane per i versi successivi. 
Per tali ragioni ritengo di proporre, per il v. 2, la seguente alternativa: TES 2 demente luce tramortita.

Abbiamo qui un accettabile novenario, reso instabile — "assurdo," se a tutti i costi così si vuole - dallo spostamento del secondo accento dalla tradizionale quinta sillaba alla quarta.

Verso 3.

Abbiamo qui necessità di trovare un equivalente per "Живи еще хоть четверть века," "Vivessi tu anche un altro quarto di secolo ..." Nella loro sistematica de-prioritizzazione (o, detto apertis verbis: soppressione, abolizione) di ritmo e rima, Ripellino e Carnevali scrivono le due seguenti meraviglie alate:

RIP 3 "Pur se ancora vivrai venticinque anni";

CAR 3 "Vivessi ancora un quarto di secolo."

Pescatori per parte sua propone: "E per quanti anni la sorte ti dia —," che se non altro rima con la "farmacia" del suo v. 1; è già qualcosa.

Che fare? Certo, in un mondo ideale l'espressione che cerchiamo dovrebbe effettivamente rimare con la parola "farmacia" in chiusa al verso 1. A questo scopo propongo di manipolare lievemente i numeri di Blok: trasformando i suoi 25 in "trenta" (ma "venti" funzionerebbe altrettanto bene sotto ogni aspetto), possiamo creare l'endecasillabo italiano seguente:

\section{TES 3 Trentanni avessi ancora in vita mia}

Verso 4.

Per il verso 4, la soluzione di Ripellino è "sarà sempre così. Non c'è rimedio." Carnevali: "non cambierebbe nulla. Senza scampo." Trovo sorprendente la riluttanza di entrambi ad usare la semplice parola uscita per is-xod. Ghini ha perfettamente ragione (comunicazione privata all'autore) nell'indicare un possibile parallelismo con il biblico exodus. Tuttavia, ancor oggi in greco - al di fuori del sensus technicus acquisito nei libri sacri - $-\varepsilon \xi-$ oঠoৎ significa né più né meno del suo calco latino "ex-itus": vale a dire, appunto, "uscita." Quest'ultima è appunto la migliorìa introdotta da Pescatori: "così per sempre, senza uscita."

Quanto sin qui detto spiega la mia proposta alternativa: 
Questo verso ha dieci sillabe, ma i suoi accenti sono distribuiti in modo tale da renderlo, più che un decasillabo, un endecasillabo con aferesi iniziale.

Nella seconda metà della poesia il poeta si rivolge al proprio tormentato io lirico, facendone un "tu lirico": "Morirai, e inizierai di nuovo dall'inizio." Ripellino traduce: "Tu morirai - comincerai di nuovo"; e Carnevali: "Muori e rinasci ancora." Pescatori: "Morrai; poi ricomincerai daccapo."

Ripellino usa il tempo futuro (e come lui Pescatori), dimostrandosi, come spesso avviene, più preciso di Carnevali, anche se forse un pochino più pedante; in ogni modo il presente storico italiano può sostituire il futuro immediato. Ma il vero problema di queste tre versioni - questioni di ritmo a parte - è che "di nuovo," "ancora" e "daccapo" ci mettono in un ginepraio dal punto di vista della rima a venire: la quale, con tali scelte, non potrà mai venire, e difatti non verrà.

Tenendo conto di tutto ciò, propongo la soluzione

TES 5 Muori - ricomincerai tal quale

Come il mio verso 4, così anche il mio verso 5 è un endecasillabo che ha perso la prima sillaba; è, per così dire, un endecasillabo in incognito. Dire "tal quale" per rendere "опять сначала" presenta un costo semantico non particolarmente alto, avendo per contro un vantaggio specifico che diverrà presto evidente.

Verso 6.

Il verso 6 di Blok, "e si ripeterà tutto come in passato," non crea problemi di interpretazione. Ripellino lo rende con "e tutto riaccadrà come una volta"; Carnevali con "e tutto sarà uguale come prima." Pescatori essenzialmente ripete Carnevali con "e tutto sarà ancora uguale" — perché mira alla rima con il suo proprio "fanale" finale (si licet il doppio bisticcio). Ora, a chi scrive pare alquanto strano che qui nessuno dei tre traduttori post-poggioliani abbia voluto seguire la strada semantica fino in fondo, come per coerenza si sarebbe dovuto, scrivendo "tutto si ripeterà." Il verbo повториться significa infatti "ripetersi": senza se e senza ma. Da dove viene questa strana distrazione "all'unisono"?

A parte il non distrarsi, nel verso 6 la vera sfida per il traduttore diligente consiste nel trovare una formulazione italiana che - oltre a dare una sequenza di sillabe accettabilmente ritmica - prepari anche il terreno per una rima compatibile con quella che sarà presto richiesta dalla fine del verso 8 di Blok, "fonar'." E poiché, nel verso 1, ho tradotto "fonar"” con 
"lampione," ecco che per quanto mi riguarda propongo come traduzione del v. 6 il seguente endecasillabo puro e semplice:

TES 6

e tutto tornerà, senza eccezione:

Una tale proposta ha il pregio di: I), essere semanticamente vicina a Blok, e II), sciogliere in anticipo ogni nodo rispetto alla necessità di far rima con il verso 8 , come vedremo.

\section{Versi 7 e 8.}

Ed ora eccoci ai versi 7 ed 8, che consistono nella sequenza di nomi suaccennati: le apposizioni, nonché esemplificazioni, del ricorrente vsë, "tutto."

Per i versi 7, "la notte, il ghiacciato incresparsi del canale," e 8, "la farmacia, la via, il lampione," Ripellino scrive: "gelido incresparsi del canale, / notte, farmacia, strada, fanale" — due bellissimi decasillabi in rima baciata! Per parte sua, Carnevali toglie la parola "notte" dall'inizio del verso 7 , andando poi a sporgerla - e facendola penzolare fuori da ogni schema semantico o ritmico - all'estremità finale di quel verso medesimo. Carnevali ottiene così "7 freddo incresparsi del canale, notte, / 8 strada, fanale, farmacia" (senza rime di alcun genere; in questo, Carnevali è coerente con se stesso).

Pescatori scrive poi: "7 notte, un brivido sul canale gelato, / 8 farmacia, strada, fanale" - formulazione che non ha ritmo, naturalmente, ma almeno ha delle rime, o quantomeno ne ha una (uguale / fanale; invece, daccapo / gelato risulta essere accoppiamento problematico sotto molti aspetti, su cui non mi dilungo).

Viste le tante soluzioni estreme proposte per questi due versi, non sembrerà forse fuori luogo che io suggerisca l'alternativa seguente come potenziale esempio di un più tranquillo compromesso:

\section{TES 7 la notte, il gelido incresparsi del canale, \\ TES $8 \quad$ la farmacia, la strada, ed il lampione.}

Questi due versi costituiscono una resa semantica esatta rispetto a Blok; e di Blok mantengono anche lo schema di rime.

Dal punto di vista ritmico, TES 8 è un endecasillabo; avrei voluto che lo fosse anche TES 7 - e avrei potuto crearne uno, anche abbastanza levigato, eliminando la parola "la notte" - ma il verso originale contiene troppo materiale semantico assolutamente vitale perché osassi compiere un tale atto di lesa maestà. E dunque, nella mia versione "la notte" rimane in capo al verso 7; cosa che rende quest' ultimo un endecasillabo aumentato da due 
sillabe in anacrusi. Se i russi possono esprimere in 9 sillabe immagini che in italiano ne richiedono 13 ... ebbene, tant'è. Come gli economisti, o gli amministratori, i traduttori non possono prioritizzare tutto contemporaneamente; e quando la fortuna li abbandona, li abbandona. Soffrono della condizione umana i poeti; e chi sono i traduttori per volersi ribellare alla propria?

\section{Dall'atelier del traduttore. Versione complessiva}

Ecco dunque una versione complessiva dei miei suggerimenti per una nuova versione italiana della seconda "Danza macabra" di Blok:

\begin{tabular}{|c|c|}
\hline $\begin{array}{l}\text { TES } 1 \\
\text { TES } 2\end{array}$ & $\begin{array}{l}\text { Notte, strada, lampione, farmacia, } \\
\text { demente luce tramortita. }\end{array}$ \\
\hline TES 3 & Trent'anni avessi ancora in vita mia, \\
\hline TES 4 & tutto sarà eguale. Non c’è uscita. \\
\hline TES 5 & Muori - ricomincerai tal quale, \\
\hline TFS 6 & e tutto tornerà, senza eccezione: \\
\hline TES 7 & la notte, il gelido incresparsi del canale, \\
\hline TES 8 & la farmacia, la strada, ed il lampione. \\
\hline
\end{tabular}

\section{Considerazioni conclusive:} Per procedere oltre la traduzione monodimensionale

Ritorniamo ora, per una riflessione conclusiva, ad un livello generale e teorico del discorso. Tutte e quattro le preesistenti traduzioni blokiane qui esaminate denudano il nucleo del nostro dilemma di partenza: che cosa deve tradurre un traduttore?

Omero si può tradurre semanticamente - certo, con più o meno arte - perché la sua poesia è sostenuta da un intreccio narrativo: ivi sono molte cose che "succedono." Ma nel testo lirico, e soprattutto nel testo simbolista, che cosa "succede"? Nel testo lirico, e soprattutto nel testo simbolista, di fatto, succedono soprattutto (non dico soltanto) ritmi e rime. Tolti quelli, al traduttore (al traduttore monodimensionale) non resta che rassegnarsi a voltare una serie di immagini da un idioma fortemente connesse in un altro idioma che fortemente le sconnette. All'estremo, per tradurre così non è nemmeno necessario che il traduttore monodimensionale comprenda qual è il legame che lega tra loro quelle parole nel testo d'origine: se quel filo andrà comunque perso nel tragitto, perché scervellarsi a cercarlo prima di partire? ... E, talvolta, chi legge ha con rammarico la sensazione che, 
appunto, esso potrebbe non essere stato cercato affatto ${ }^{14}$.

Come si presenta invece la sfida per chi desideri tentare di far meglio ${ }^{15}$ ?

Per il traduttore multidimensionale, per il traduttore poetico, che si sente a disagio all'idea di produrre "bigini semantici" o traduzioni "di servizio" dell'originale (incautamente?) affidatogli, vuoi dall'editore, vuoi da un inscrutabile destino, la sfida - soprattutto la sfida presentata da testi fatti di "musica in parole" qual è la poesia simbolista — è di tradurre non parola per parola, ma immagine per immagine, includendo nell'opera ricreata tutte le componenti del testo d'origine: anche il loro tessuto materiale, non soltanto la loro "prosa." Insomma, il traduttore multidimensionale è ben conscio dell'aforisma di Briusov (e fa del suo meglio per agire di conseguenza) secondo cui, com'è noto, "i versi si scrivono al fine di dire più di quanto si possa dire in prosa" 16 .

Un poeta lirico, e soprattutto un poeta simbolista, pedissequamente tradotto fa dire al lettore della traduzione: Ma che farneticava quel pazzo?, allorquando chi legge l'originale esclama: Sì è così; non poteva essere altrimenti, poiché qui tutto si tiene ${ }^{17}$.

È quando il lettore vede l'originale, che il traduttore è riuscito a dire.

\section{University of British Columbia, Vancouver}

14 Checché se ne dica, la "Gretchenfrage" (mi spiego: la quaestio ingenua che ha però il pregio di andare dritto all'essenziale, chiamando pane il pane e vino il vino - o un chat un chat, o a spade a spade, a seconda), ruota comunque intorno alla ricerca "nach der 'besseren' oder 'schlechteren' Übersetzung”, "della traduzione 'migliore' o rispettivamente 'peggiore". "Was ja nichts anderes heißt, daß eine derartige Frage auf der Metaebene diskutiert wird, d.h. hier auf der Ebene der Interpretation (einsprachig) und nicht in der zweisprachigen Beziehung Ausgangssprache-Zielsprache”. Pogatschnigg 401.

15 [T] he role of the translator is central. The measurable importance of semiotic structures notwithstanding, the style and talents of the individual translator will always play a key role in shaping the translated text. With regard to inspirational translation, this is a matter of course, but even within conventionalized translation, this remains a fact. Gottlieb 26.

16 Стихи пишутся затем, чтобы сказать больше, чем можно в прозе. Cit. in Lotman 1968 [1964]: 186.

17 Con più autorità del sottoscritto già si espresse in tal senso un traduttore professionale del mondo antico: "Si ad verbum interpretor, absurde resonant." Hieronimus 1980: 14, q.v. per una trattazione più dettagliata. 


\section{OPERE CITATE}

Atti del $9^{\circ}$ Convegno sui problemi della traduzione letteraria e scientifica. Le traduzioni dal russo. A cura dell'Amministrazione Comunale. Vol. 10 (1981). Monselice (Padova): 1981.

Blok, Aleksandr. Polnoe sobranie stixotvorenii v dvux tomax. A cura di Vladimir Orlov. Mosca e Leningrado: Sovetskii pisatel', 1946. 2 vol. (Biblioteka poèta. Coordinatore: Yurii Nikolaevič Tynianov). Citato come PSS.

—. Poesie. Introd. e trad. di Bruno Carnevali. Roma: Newton Compton, 1977. (Paperbacks poeti 52). [Edizione bilingue; 77 poesie di Blok.]

—. Poesie. Introd. e trad. di Angelo Maria Ripellino. Milano: SE Studio Editoriale, 1987 [1975]. (L'altra biblioteca). [Soltanto trad. italiana senza originale; 131 poesie di Blok, coincidenti con quelle della ed. Guanda qui infra.] Poesie. Introd. e trad. di Angelo Maria Ripellino. Prefaz. Valerio Magrelli. Parma: Guanda, 2000 [1975]. (Poeti della Fenice). [Edizione bilingue; 131 poesie di Blok, coincidenti con quelle della succitata ed. SE $1987+$ testo russo a fronte.]

Brecht, Bertolt. Gedichte 4. In Werke. Berliner u. Frankfurter Ausgabe. A cura di Werner Hecht et al. Vol. 14. Berlin: Aufbau e Ff/M.: Suhrkamp, 1988.

Brik, Osip. "Ritm i sintaksis" (1927). In italiano: in I formalisti russi. A cura di Tsvetan Todorov. Torino: Einaudi, 1968 (PBE 111). 151-85. In inglese: in Readings in Russian Poetics. A cura di Ladislav Matejka e Krystyna Pomorska. Ann Arbor, Mich.: Michigan Slavic Publications, 1978. 117-25.

Buffoni, Franco. La traduzione del testo poetico. Milano: Guerini, 1989.

Cucchi, Maurizio. "Sulla deperibilità del testo poetico tradotto." In Buffoni 1989. 93-95.

Gerzymisch-Arbogast, Heidrun, e Sandra Nauert, a cura di. Challenges of Multidimensional Translation. Proceedings of the Marie Curie Euroconferences. Saarbrücken 2-6 May 2005. http://www.translationconcepts.org/pdf/ MuTra_2005_Proceedings.pdf\#page=5

Gerzymisch-Arbogast, Heidrun. "Introducing Multidimensional Translation." In Gerzymisch-Arbogast 2005. 1-15.

Ghini, Giuseppe. "Tradurre il ritmo del poeta: Pushkin nelle 'versioni ritmiche' di Poggioli." Studi Slavistici 2 (2005). 81-96.

Giudici, Giovanni. "Da un’officina di traduzioni.” In Buffoni 1989. 81-91.

Gottlieb, Henrik. "Multidimensional Translation: Semantics Turned Semiotics." In Gerzymisch-Arbogast 2005. 1-29.

Hieronimus. Liber de optimo genere interpretandi (Epistula 57). Ed. G. J. M. Bartelink. Leiden: Brill, 1980.

Hölderlin, Friedrich. Le liriche. Trad. Enzo Mandruzzato. Milano: Adelphi, 2004 [1977].

Jakobson, Roman. “On Linguistic Aspects of Translation.” In Venuti 2000. 113-18. Levý, Jiří. “Translation as a Decision Process." In Venuti 2000. 148-59.

Lotman, Iurii Mikhailovič. Analiz poètičeskogo teksta. Struktura stixa. Leningrad: Prosveščenie, 1972. In inglese: Analysis of the Poetic Text. Ed., trans. D. Barton 
Johnson. Ann Arbor, Mich.: Ardis, 1976.

—. Lektsii po struktural'noi poètike. 1964. Ristampa anastatica: Intro. Thomas G. Winner. Providence, R.I.: Brown University Press, 1968.

Masseau, Paola. Une traductologie de la poésie est-elle possible? Paul Valéry et la traduction du poème "toujours recommencée». Paris: Editions Publibook Université (EPU), 2012.

Osimo, Bruno. Il manuale del traduttore. Milano: Hoepli, 1998.

Pescatori, Sergio. "I lampioni sono poetici?" In Atti [...] Città di Monselice 1981. 73-89.

Pogatschnigg, Gustav-Adolf. "Mikrostruktur und Makrostruktur. Ein Beitrag zur Theorie der Übersetzung." In Buffoni 1989. 395-404.

Poggioli, Renato. Il fiore del verso russo. Torino: Einaudi, 1949.

Ramous, Mario. "Il pretesto della poesia (di Catullo e altro)." In Buffoni 1989. 225-34.

Sansone, Giuseppe. "Traduzione ritmica e traduzione metrica." In Buffoni 1989. 13-28.

Sosio, Libero. "Brutte e infedeli. Noterelle sul lavoro di traduzione." In Atti [...] Città di Monselice 1981. xxxix-xlix.

Testa, Carlo. “Quali poeti ha letto l'Italia del ventesimo secolo? Rovello in un prologo e 9 documenti: Blok, Akhmatova, Mandel'shtam, Pasternak." Italian Poetry Review 3 (2008). 207-27.

Venuti, Lawrence. The Translation Studies Reader. Londra e New York: Routledge, 2000. 